\title{
PARA UN ESTUDIO DE LA CREATIVIDAD LÉXICA DE RAMÓN GÓMEZ DE LA SERNA
}

En un estudio que aparece este año conmemorativo del centenario del nacimiento de Gómez de la Serna en un número monográfico de la Revista de Occidente a él dedicado*, dijimos que en la escala de posibilidades de innovación y creatividad con el lenguaje existían dos vertientes, ascendente y descendente, en las que, a medida que se avanzaba en la complejidad (hacia arriba) o en la simplificación (por debajo del nivel morfemático), disminuían las potencialidades del hablante para innovar con voluntad consciente. Entre ambas queda, pues, el espacio de mayor creatividad, en torno al lexema y la frase. No será de extrañar que sea en este espacio donde la abundancia y originalidad de la vena creadora ramoniana se manifiesten con mayor fuerza, aunque quizá no tan espectacularmente como en los géneros literarios.

No habrá que sorprenderse si las características innovadoras en el nivel frástico y en el léxico se revelan en Gómez de la Serna perfectamente acordes con su proyecto revolucionario en sus dos caras destructora y reconstructiva. Una reconstrucción, dicho sea de paso, que rehúye previsoramente toda planificación armónica, equilibrada, clasicizante, y que persigue insistentemente el mantenimiento de la espontaneidad, la esponjosidad y el carácter improvisador del bricolage - diríamos chapucería si el lector aceptase positivizar no sólo las connotaciones sino la denotación del vocablo castellano.

En el nivel frástico, los estudiosos de Ramón han venido coincidiendo, a partir de una acomodación a la postura normativista, académica, en cuestiones gramaticales, para hablar -como también se hizo en su día con Pío Baroja- de un faprestismo que se manifestaría en toda clase de incoherencias ("incongruencias" y

* "El gato encerrado", ROcc, 1988, núm. 80, 31-62. 
"absurdos" hubieran sido términos aceptados por Ramón) resumibles en el muy frecuente fenómeno del anacoluto. Es éste un achaque reprochado al escritor fundándose en su capacidad (frente al hablante) de releer su texto y corregir las incoherencias originadas, resultantes de los desfases entre la velocidad de construcción mental del discurso, de su transcripción en frases, y de éstas en signos gráficos. Igualmente se ha observado en Gómez de la Serna un absoluto descuido de la corrección ortográfica, tanto para la transcripción de palabras extranjeras como para la del castellano en general. Creemos que estas cuestiones, como cualesquiera otras, deben primeramente considerarse en función de la programática teórica de los creadores literarios, si éstos, como es el caso de Ramón, se han manifestado explícitamente a su respecto.

Dejando de lado la cuestión de la ortografía castellana, que se acabó resolviendo, en el caso de Ramón, como en el de tantos otros ilustres escritores y académicos, con la intervención experta de tipógrafos y correctores de pruebas, el "descuido" de su sintaxis está explicado y razonado - así lo creemos - como una faceta más del combate contra el statu quo y el anquilosamiento academicista. Esta ligereza en la construcción responde al ya subrayado principio ramoniano de la distensión, de la creación de espacio intersticial, aireando la prosa - como los contenidos- del amazacotamiento y falta de porosidad en la prosa lamida y relamida, visada y revisada, que a fuerza de tales manejos gana en corrección y equilibrio cuanto pierde en espontaneidad y en turbulencia, alejándose así de la realidad cotidiana. A ella buscaba ajustarse Ramón con una prosa espontaneísta, restallante de vitalidad e indiferente, mejor aún, enemiga de esa preocupación monumentalizante de escribir para la eternidad.

Esta justificación, que hemos dado siempre para explicar las características sintácticas de la prosa ramoniana, está confirmada no sólo por su coherencia con todo su programa revolucionario (primero ético-estético, luego únicamente estético), sino también por algunas afirmaciones programáticas muy concretas y convincentes, hoy prácticamente ignoradas por los estudiosos de la obra ramoniana a partir de las versiones expurgadas posteriores a 1939. En Mis siete palabras (1910) y, sobre todo, en el Ex-libris de El Rastro (1914), se encuentra una justificación que confirma la manifiesta voluntad del joven escritor de dejar sus textos en la forma primigenia de la primera redacción. Son particularmente definitivas las afirmaciones de las pp. 258-264, de las que extraemos algún párrafo: 
Ha de notarse en un libro, sin arrepentirse después de haberlo hecho, ha de notarse, ha de verse en él, la mala gana, la negligencia de emplear el estilo prostituido, la lengua de todos que nos ha repugnado aun en sus frases más preciosas. ¡Cuánta intención en no tropezar, en no incurrir en ciertas frases! y eso ¡cuántos zis-zas y cuántas quebraduras y cuántas torpezas produce a veces! ¿Cómo o por qué explicar esos líos inevitables, esas incipiencias en el estilo? ¿Esta defección de la prosa, este no corregir las transposiciones visibles, esta repugnancia a distanciar con requilorios accidentales las palabras difíciles que de pronto se reúnen con acritud, con una pasión violenta y aislada, con cierto esquinamiento, todo esto no es necesario ante lo penoso que es encerrar en frialdad, en un cuido artificial de fabricantes, lo que se produce a sí mismo? ¿Es que no es contrahecha toda geometría perfecta, excesiva, formal?... ¿Es que se debe volver sobre la materialidad de la oración gramatical como tal oración? Ese volver atrás descompone, quiérase o no, todo el ensimismamiento con que habíamos concebido, olvidados de la oración en favor de la palabra. ¿Es que no es mejor para tapar un vacío una palabra sugeridora aun bajo un aspecto de absoluta improcedencia que cubrir ese vacío con una palabra reticente y ambigua? ¿Es que esa afonía que se nota a veces en un estilo no es más reveladora que la plena voz impasible? ¿Cómo o por qué explicar que no se ha podido por infinitas razones de sencillez ser más sencillo y que hasta se ha intentado hacer más balbucientes los balbuceos? La perfección del balbuceo, el no avergonzarse ni corregir el balbuceo, es la única forma admirable de expresión, es la única manera de que haya potencialidad en el estilo. Esa misma tribulación de la prosa es necesaria, y así se dice lo que de otro modo más desbrozado y correcto no se diría. Hay que sacrificar el estilo a lo que es superior a él, a la ansiedad, que si bien es de apariencia defectuosa, deplorable y quejosa, es el único motivo artístico considerable, hemos de ser incondicionales de la ansiedad desnuda a la que cualquier cuidado convencional del estilo oculta tan inmodificablemente, tan irreparablemente que después, cuando queremos volver sobre nuestra corrección de timoratos, ya no la podemos hallar tan inmediata.

Este párrafo ramoniano nos lleva de la mano a lo que, desde siempre, se evidencia en la lectura de la obra del polígrafo madrileño: la abundancia neológica que no retrocede, concorde con su programa revolucionario, ni ante las cortapisas académicas que abominan del barbarismo, entendiendo por tal no sólo la pronunciación, escritura o uso "incorrectos" de las palabras, sino la neología "innecesaria". Se juzga por tal la que considera que en el caudal léxico castellano existen inventariadas ya las palabras "ade- 
cuadas" para una determinada designación. La "adulteración", por descontado, es juzgada como más grave si el neologismo es una reproducción o adaptación más o menos cruda de una palabra de otra lengua. Está claro que esta producción incontenible de neologismos entraba perfectamente en el programa revolucionario ramoniano. De la mezquina incoherencia de tal norma academicista da fe el hecho de que galicismo o anglicismo sean considerados como violaciones de la "pureza" del idioma, mientras que los arabismos son timbres de la gloriosa identidad de la lengua española. Ya en 1909, citando de memoria a Larra, recordaba Ramón: "A las palabras no hay que preguntarlas ¿de dónde vienes? sino ¿para qué sirves?"

Desde sus primeras hasta sus últimas obras, Ramón conservaría una asombrosa capacidad neologizante, que aún no puede valorarse en su totalidad por una simple razón. En castellano no disponemos - a diferencia de otras grandes lenguas de comunicación occidentales - de un diccionario tesaurizante, es decir, con voluntad de inventariar todas las palabras utilizadas en el transcurso de la historia de la lengua, desde sus orígenes hasta nuestros días, sin discriminar ninguna por ningún tipo de razones. El seminario de lexicografía de la Real Academia Española viene realizando, a partir de un inventario documental muy deficiente para la época contemporánea y para el área hispanoamericana en particular, una primera obra de este tipo, titulada Diccionario histórico de la lengua española, pero hasta la fecha sólo lleva realizados y publicados diez y siete fascículos inventariando desde la palabra $a$ hasta la palabra anafrodítico. Mientras tal labor, iniciada su publicación en 1960, no se complete (y aun si se completase, y a causa de las graves deficiencias de su inventario) no es posible una evaluación adecuada y precisa de la importancia relativa de la creatividad léxica de Ramón, a falta de poderla comparar con la de los demás escritores y con la del conjunto. Por el momento, sólo en algunos aspectos y muy parcialmente se la puede comparar con la de otros contemporáneos como Valle-Inclán, sobre cuyo léxico se han producido algunas monografías y tesis inéditas, o como García Lorca, de cuyo vocabulario poseemos un inventario bruto, realizado con ayuda del ordenador, y disponible en forma de concordancias. De esta manera, sólo el aspecto de la neología pura, es decir, de la creación de palabras nuevas, puede examinarse, y aun con una importante precaución. En efecto, si una palabra documentada en un texto ramoniano de 1910 no aparece en ningún diccionario de fecha anterior, hay probabilidades de 
que sea creación suya, pero mientras el despojo documental sea tan deficiente como hasta ahora, sigue siendo posible que Ramón no sea su creador o adaptador. Con todas estas y muchas otras salvedades, y reconociendo el inevitable cariz impresionista de nuestras apreciaciones, intentaremos una evaluación provisional de la creatividad ramoniana.

En primer lugar, en el nivel léxico, nos limitaremos a evaluar el acervo léxico ramoniano para las palabras incluidas alfabéticamente entre $a$ y anafrodítico, únicas que pueden compararse con el estudio del Dicc. hist. Por lo que toca al léxico de Ramón, el Dicc. hist. ha utilizado un número selecto y reducido de textos (14 obras hasta 1979, fecha de edición del fascículo 14, de alito a aloja, a las que se han sumado otras dos entre 1979-1981, y una entre 1981 y 1986). Estos textos no son siempre primeras ediciones, y hay incluso errores graves de datación. Así se da a Tapiccs (colección de textos publicados entre 1911-1912, y editados en libro en 1913) la fecha de 1922. Se da como fecha de creación de El circo ca. 1924, cuando la primera edición es de 1917. No es el Dicc. hist. documento suficiente para valorar el léxico ramoniano, como lo es, sin duda, para el de algunos de nuestros clásicos como Cervantes o Lope de Vega. Pero es evidente, aun así, que no son infrecuentes en él las entradas canónicas de palabras documentadas únicamente en Gómez de la Serna. Allí aparecen, pues, como hapax legomenon, neologismos significativos de 1910-1911, como absurdizar, absurdidad y absurdizable. En nuestras lecturas de la obra ramoniana hemos recogido ochenta y cuatro neologismos que no se documentan en el Dicc. hist. Nótese que nuestra lectura de dicha obra, por atenta que haya podido ser, no deja de tener resultados azarosos, puesto que no establece un inventario exhaustivo de su vocabulario, ni compara palabra por palabra con el inventario del Dicc. hist. Se fía únicamente de la imprcsión de extrañeza que le causa la presencia de determinadas palabras, extrañeza resultante de su propia experiencia como lector. Por eso, y paradójicamente, la incapacidad para extraer impresionísticamente las palabras inusuales en la lectura aumenta con la cultura del lector y con su propia disposición para la creatividad léxica, justificándose así definitivamente la extracción de inventarios exhaustivos y sus cotejos por medio de ordenadores con un inventario total.

De las lecturas realizadas en años precedentes teníamos recogidos 60 neologismos que ahora, con la relectura de textos exigida por nuestro trabajo, se han incrementado en número notable -24- que, sin duda, seguirá creciendo en futuras lecturas, lo 
que prueba, si falta hiciera, que una empresa lexicográfica con las pretensiones de la del Dicc. hist. no puede prescindir de la ayuda de ordenadores y de la lectura automática de textos. Hasta que esto se realice, nuestro conocimiento del léxico hispánico será de una tremenda imprecisión. Aun descontando que es prácticamente imposible el inventario total de un corpus en perpetua mutación, los cálculos estadísticos pueden dar con gran precisión unos índices de frecuencia de uso del léxico válidos a partir de un mínimo determinable que, de cualquier modo, es, para el castellano hispánico, muy superior a los diez millones de datos léxicos (ocurrencias, en términos lexicográficos) de que se dispone para el Dicc. hist. Pero hace falta un volumen aún mayor de datos para alcanzar un porcentaje aceptable de los neologismos producidos, no ya en el habla cotidiana de trescientos millones de castellanohablantes, ni siquiera en la prensa periódica y la radio-televisión, sino simplemente en la creación textual considerada digna de recordación por la institución literaria.

En nuestra recogida de palabras inusuales en la obra de Gómez de la Serna hemos encontrado también un número considerable de voces y acepciones - 42- que en el Dicc. hist. aparecen como de uso exclusivo de otros escritores anteriores, coetáneos o posteriores a él. Esta abundante resolución de supuestos hapax resulta evidenciadora también de la imprecisión y la provisionalidad de la información disponible en el Dicc. hist., con ser, como es, indiscutiblemente, un paso adelante enorme en la historiografía de la lengua, que nos situará, si se completa a un ritmo mucho más rápido que hasta ahora, dentro de unos cien años, a la altura del diccionario de Oxford para el inglés, que se completó en los años treinta, pero a mucha distancia aún de los diccionarios basados en corpus como el del TLF francés o el diccionario de la Crusca italiana, que cuentan con una infraestructura de tratamiento de datos por ordenador.

No se limita la creatividad léxica de Ramón a la plasmación de neologismos, con ser ésta la más espectacular de sus facetas. Es aún más abundante la adaptación de palabras ya existentes a significados novedosos, exigidos por su personalísima visión de la realidad. No es sorprendente, pues, que a los inventariados por el Dicc. hist. hayamos podido añadir 115 casos más de acepciones nuevas de palabras. Teniendo en cuenta la precariedad de la información contenida en el Dicc. hist., los siguientes datos sobre los usos léxicos de Gómez de la Serna deben tomarse cum grano salis. Los 33 casos inventariados por nosotros (siempre de pala- 
bras comprendidas entre $a$ y anafrodítico) en la obra de Ramón, además de los que constan en el Dicc. hist., y en los que una palabra documentada por primera vez en América la vemos testimoniada por primera vez en España, con posterioridad, en él, ¿'es prueba realmente suficiente de que haya sido un fervoroso lector y aceptador de la neología castellana de América? El hecho de que en nuestra documentación aparezcan más de 40 palabras usadas por primera vez por Ramón, aunque ya estén documentadas anteriormente en diccionarios no académicos (Zerolo, Domínguez, etc.), ¿es índice de que Ramón recurría a dichos textos lexicográficos para incrementar su vocabulario activo, o es simplemente resultado de carencias documentales, y habrían sido ya usadas anteriormente a él por otros creadores literarios?

El Dice. hist. , cuando define cada acepción de una palabra, la acompaña de un número de ejemplos de uso que considera suficientes, por orden cronológico y citando obligatoriamente el primer y el último ejemplo que constan en su documentación. La información se completa con un paréntesis del tipo: $(+12$, ss. XVIII-Xx) que indica que, además de los citados expresamente, quedan en el inventario académico doce ejemplos no citados, fechados entre ambos siglos. Si no hay sobrante, por descontado, tal referencia desaparece. Pues bien, el hecho de que conste en nuestra documentación un número de 161 palabras o acepciones de palabras usadas por Ramón y para las cuales el Dice. hist. no da ningún sobrante para el siglo $\mathrm{xx}$, ¿es índice suficiente de que a Ramón le gustaba utilizar palabras inusuales y envejecidas, o sólo es un reflejo más de las deficiencias documentales del Dice. hist.? Si nos detenemos en rarezas como absintio, absolutivo, abismático, ajadura o almo, cabe pensar en lo primero. Pero hay que pensar en deficiencia documental cuando se encuentran adjetivos como abrileño, abombado, abusivo, acaracolado, aclarador, aconchado, acordeonado, adivinador, agosteño, ajedrezado, alfombrado, aliñado ( $1^{\mathrm{a}}$ acepción), nombres como abisinio, abombamiento, abracadabra, abultamiento (acep. 2), aburrición, aceite de hígado de bacalao, aceite de ricino, acémila (acep. 3), acento circunflejo, acuarelista, adivinador, agresión, agua de azahar, aguadera, aguafortista, alambrera (de brasero), albañilería, albérchigo, alumbrado, o verbos como abarquillar, abovedar (acep. 2), absolver (acep. 8), abultar (acep. 5), acotar, achular, aderezar ('condimentar'), agravar (aceps. 5 y 7) o alambicar (acep. 1).

Asimismo, ¿qué quiere decir que, considerando nuestra documentación sobre Ramón, se adelante en más de 60 palabras o acepciones de palabras la datación del primer testimonio de uso 
documentado en el Dicc. hist.? ¿Que Ramón creó otras tantas voces o acepciones nuevas usadas luego por otros escritores, o que, de nuevo, la documentación es insuficiente? En el primer caso, sería un buen indicio de la aceptación que las innovaciones léxicas ramonianas tuvieron en la comunidad literaria, y, por consiguiente, un dato fundamental para la historia de su recepción. Pero no conviene darlo por sentado mientras no podamos consultar un corpus de, al menos, cien millones de datos léxicos. ¿Qué quiere decir que, según nuestro fichero, 156 palabras y acepciones de palabras estén documentadas en Ramón con fechas posteriores a la última documentada por el Dicc. hist., y a distancias de ésta que varían entre los tres siglos y los diez años? Cuanto mayor sea el desfase cronológico, mayor probabilidad existe de que sean datos indicativos de un gusto arcaizante en Ramón. Pero los casos documentados sólo excepcionalmente llegan a tales lapsos, y generalmente no se trata de palabras sino de acepciones en desuso de palabras usuales como alisar, cuya acepción $1 \mathrm{~b}$ del Dicc. hist. no se documenta ahí desde el siglo xvII, y que podría bien ser una simple recreación ramoniana. En la medida en que la distancia disminuye (y la mayoría de los casos no sobrepasa el siglo), más bien habría que pensar en deficiencias del Dicc. hist. ya que, por vocación explícita, Ramón era alérgico a los valores establecidos y al principio de autoridad, fuese ésta la emanada del Ministerio de Orden Público (en su movida juventud) o la de los cinco tomos del Diccionario de Autoridades de la Academia dieciochesca.

A la misma necesidad expresiva de Ramón corresponden otras tendencias en las que parece más fácil dictaminar su liderazgo, al menos en lo que a la narrativa del siglo $\mathrm{xx}$ peninsular se refiere. Nos referimos a su adopción del léxico madrileño, y no con int znción folklorista de ambientar sus textos a la realidad circunstancial de su novela, ya que la asume como narrador, además de en los diálogos de sus personajes. Suponemos que a lectores adictos de Ramón como Francisco Umbral, que son en eso fieles a la programática ramoniana, no se les habrá escapado la presencia de algún que otro "lo cual que...", expresión popular que algunos creen, o poco menos, creación del "cheli'" madrileño de los sesenta. No sólo lo hemos documentado en Ramón (Muestrario, 1919) sino que se encuentra en la prensa popular de 1908 y 1909 (El socialista de Madrid). Coloquialismos y popularismos como faltar a la madre, jorobar, esnucar, cualquieras, chisgarabis, 'golfa, ¿no, tú? los hemos encontrado en Tapices y en Mis sicte palabras (1909). 
Tal vez más indicativo del influjo que ejerció Ramón sobre la generación de 1923 es que, según nuestros ficheros léxicos reunidos para un estudio sobre la prosa vanguardista, no menos de 35 neologismos ramonianos aparezcan documentados posteriormente en Antonio Espina, Jorge Guillén, F. García Lorca, B. Jarnés, V. Andrés Álvarez, M. Fernández Almagro, J. J. Domenchina, Max Aub, E. Giménez Caballero, etcétera.

De toda la actividad renovadora del lenguaje por parte de Gómez de la Serna, es posiblemente el aspecto estilístico que hemos denominado "desplazamiento figurativo total y circular" lo que más ha marcado la historia de la prosa vanguardista, pero, evidentemente, sobrepasa los límites de este trabajo dedicado a la creatividad léxica. Enviamos al curioso lector al artículo titulado "El gato encerrado" al que hacíamos referencia al comienzo de estas páginas.

\title{
Ignacio Soldevila-Durante Université Laval, Quebec
}

\begin{abstract}
APÉNDICE
Acompañamos este trabajo con listas documentales que avalan los asertos más importantes. Hemos prescindido de documentar las afirmaciones más eviden-tes, como las documentaciones posteriores a la última datación del Dicc. hist., pero están nuestros archivos disponibles para la consulta del curioso lector. Se notará que bastantes ejemplos de los citados proceden de obras de Ramón que figuran en la nómina de obras citadas en el Dicc. hist. Esto confirma, si hiciera falta, que los despojos aleatorios de los textos son inaceptables en una perspectiva científica, si el propósito es, como ocurre con el Dicc. hist., tesaurizante.
\end{abstract}

\section{Neologismos no registrados en el Dicc. hist. ${ }^{*}$}

abismamiento (1914), $E R^{* *}, 10$.

ablacionar (1918-1924), Pombo, OC, t. 2, 213.

* Si disponemos de más de un ejemplo, damos únicamente la fecha del más antiguo. Nótese que en algunos casos para el adjetivo de origen participial, la fluctuación de criterios en el Dice. hist. hace que, a veces, se integren en el verbo formas de uso adjetivo. (A este respecto, el criterio parece variar a partir del noveno fascículo, al asumir la dirección R. Lapesa.)

** Se usan las siguientes abreviaturas para las obras de Gómez de la Serna: CNL: Concepto nueva literatura; DP: Drama Palacio deshabitado; EN: El novelista; ER: El Rastro; GL: Gaceta literaria; LM: Libro mudo; LN: Libro nuevo; MO: Morbideces; OC: Obras completas; OF: Otras fantasmagorias; PI: Paisajes imaginarios; Prom: Prometeo; QP: La quinta de Palmyra; SA: Secreto acueducto; 7 palabras: Mis siete palabras; TS: El teatro en soledad; VBN: La viuda blanca y negra. Algunos textos aparecieron en la Revista de Occidente (ROcc). 
abogue (1920), $L N, 249$.

abotijamiento (1918), Muestrario, 13.

abrelibros (1918-1955), Greguerias (1915), 318; 1923, QP (1982), 173.

abreostras (1933), Ensayos heterogéneos, ROcc, núm. 116, 178.

abrepapeles (1931), Ismos, $O C$, t. 2, 1193; ROcc, núm. 95, 114.

absorbedor (1920), $L N, 46(+2)$.

abstenedor (1930-1931), Azorín, OC, t. 1, 1097.

abstraccionar (1931), Ismos, $O C, \mathrm{t} .2,1185$.

abuelete (1917), El circo, OC, t. 2, 813.

academicidad (1930), París, (1986), 194.

acarbonado, -a (1929), Hist. de Picasso, ROcc, núm. 73, 64.

accedido (1911), Moguer; (1913), Tapices, $76(+4)$.

aciagamente (1912), TS; Prom, XXXV, 11.

acidulado, -a (1908), MO, 150.

aclarativo (1912), Tristán, 162; aclaratriz (1931), Ismos, OC, t. 2, $1173(+3)$.

acodamiento (1910), Prom, 70.

acogotamiento (1912), TS; Prom, XXXVI, 21.

acoliflorado (1919), Revista España, 223, 8.

acolitismo (1918-1924), Pombo, $O C$, t. 2, 331.

acoplado, -a (1918), $V B N, O C$, t. 1, 1263.

acoquinante (1909), DP; Prom, XII, 4.

acosadoramente (1909), CNL, 20.

acosechar (1909), CNL, 18.

aculebrinar (1931), Ismos, OC, t. 2, 1209; (1923), QP (1983), 271.

achabacanado, -a (1931), Ismos, OC, t. 2, 1189.

achicaaguas (1935-1961), OF (1961), 195.

adormidor, -a (1931), La acinesia, ROcc, núm. 141, 269.

adormilamiento (1918), $V B N, O C$, t. 1, 1320.

adornito (1923), Senos, 161.

adunado, -a (1) (1916), PI (1955), Antología, 47.

adunado, -a (2) (1923), EN, 129.

adunar (de duna) (1923), $Q P, O C$, t. 1, 1597.

advenedicidad (1928), ROcc, núm. 65, 207.

afacetado, -a (1932), ROcc, núm. 107, 152.

afilación (1933), ROcc, núm. 116, 189.

aflautamiento (1912), Tristán, 176.

afrancesador (1908), Prom, I, 100.

agachapandarse (1918), $V B N, O C$, t. 1, 1339.

agiotista (adj.) (1908), $M O, 68$.

aglomerador, -a (1930-1931), Azorin, OC, t. 1, 967.

agor (1942), Epílogo a Azorín, $O C$, t. 1, 1135.

agravado, -a (1930), París (1986), 82.

agriable (1931), Ismos, OC, t. 2, 1046.

agrietado, -a (1922), S4, 194-195.

aguado, -a (1918-1924), Pombo, OC, t. 2, 95.

agudizado, -a (1930), GL, 83.

aguijado, -a (1912), TS, Prom, XXXVI, 24.

aguijonado, -a (1911), Tapices (1913), 37.

aguileñismo (1940), Mi tía Carolina, OC, t. 1, 1182. 
agujereamiento (1918), $V B N, O C$, t. 1, 1352.

ahorcador, -a (adj.) (1914), ER, 58.

ajaponesado, -a (1935-1961), OF, 144.

alaboso, -a (1917), Scnos (1955), 80.

alanceolar (1910), $L M, 122$.

alardeante (1918), VBN, $O C$, t. 1, $1314(+4)$.

alargable (1912), Tapices (1913), 24.

alarmático (1929), ROcc, núm. 69, 287.

albirrosismo (1923), Senos, 42.

alboreante (1931), Ismos, OC, t. 2, 1172.

alemanismo (1913), Ismos, $O C$, t. 2, 1129.

alemanizado, -a (1909), Prom, III, 83.

alfeñicador (1921), Doctor inverosimil (1948), 135.

alfombrilla (1923), Ramonismo, 215.

alhajado (m) (1912), Tapices (1913), 163.

alicargado, -a (1933), ROcc, núm. 116, 174.

alienado, -a (m y f) (1935), ROcc, núm. 141, 265.

alimañismo (1923), Ramonismo, 85.

aljofarado (m) (1933), ROcc, núm. 116, 176.

almadreñeo (1931), Ismos, $O C, \mathrm{t} .2,959$.

almohadillaje (1909), CNL, 17.

almohadonar (1912), Tapiccs (1913), 63.

alquimiado, -a (1930), París (1968), 113.

alquitaradamente (1909), Prom, V1Il, 102.

altimeseta (1922), ROcc, núm. 73, 65.

alzaprimante (1920), LN, 211.

amamantación (1930), París (1986), 150.

amancebación (1923), $E N, 19$.

amarguismo (1930), ROcc, núm. 84, 358.

amazonesco (1923), $Q P_{\text {( }}$ (1983), 247.

amiantar (1931), Ismos, $O C$, t. 2, 1081.

amortecinar (1930), París (1986), 73.

\section{SOlứiones De haPAX DEL DrCC. HIST}

abandonadamente (Dicc. hist.: cl501): 1908, MO, 133.

abaniquería (Dicc. hist.: 1852, Dice. Castro): 1918-1955, Pombo, OC, t. 2, 65.

abroncar (Dicc. hist.: 1927, Valle-Inclán): 1918-1924, Pombo, OC, t. 2, 127. abrumación (Dicc. hist.: 1911, Ramón): 1923, Ramonismo, 101 ( + 2, 1930, 1931). abrumadoramente (Dicc. hist.: 1939-1956 Ac.): 1920 LN, 232 (+1, 1914). acaparación (Dicc. hist.: E. Castelar) 1911, LM, $163(+1,1923)$. acople (Dicc. hist. : 1947, Ragucci): 1922, El incongrucnte, $16(+2,1948,1930)$. acrático (Dicc. hist.: 1896, R. Darío y dices.): 1948, Automoribundia, 274.

acuchillamiento (Dicc. hist., acep. 2: 1617, Oudin): 1917, El circo, OC, t. 2, $891(+1,1920)$

acusticidad (Dicc. hist.: 1954, Núñez Alonso), 1935, ROcc, núm. 141, 270. achicadero (Dicc. hist.: 1910, Cavada, voc. prov. Chiloé): 1914, ER, 68. adornismo (Dicc. hist.: 1944, Ramón): 1934, Lo cursi (1955), 188 (+ 2, 1930, 1931). 
afilacuchillos (Dice. hist.: 1962, Catálogo Zaragoza): 1933, ROcc, núm. 116, 189. afiladamente (Dice. hist.: Domínguez y Miró, 1916): 1914, ER, 151. afiladura (acep. 1) (Dicc. hist.: 1495-1877, diccionarios): 1913, Tapices, 144. agalerado, -a (Dicc. hist., como verbo, sin ejs.): 1926, Gollerías (1955), 108. agarrado (acep. 2a) (Dicc. hist. 1726 Ac.): 1920, LN, $193(+1,1923)$. agarrador (acep. $3^{\mathrm{a}}$ ) (Dicc. hist. : 2 ejs. de dice. y cédula ad hoc): 1920, $L N, 188$. agrandador (Dicc. hist.: 1919, Maura Gamazo): 1930, París (1986), 97.

agresor (Dice. hist., acep. le: 1955, Campoamor): 1935, Los muertos (1961), 37. agror (Dicc. hist., 2: 1933, Voc. andaluz): 1955, adic. a Pombo, OC, t. 2, 38. agua de agraz (Dicc. hist.: 1803-1817 Ac.): 1918-1955, Pombo, OC, t. 2, 99. aguijoneante (Dicc. hist.: 1956, Carilla): 1909 DP, epílogo, sin paginar. aislación (Dice. hist.: Tapices) 1910, La bailarina, 9; 1914, ER, 265. ajedrez (acep. 1b: Canc. Baena): 1922, SA, 123.

alcazareño (acep. 2: 1968, Manrique de Lara): 1922, SA, 107.

alemanizarse (Dicc. hist.: 1589, Pineda; 1900, Echevarría, Voces Chile): 1910,

Prom, XVI, 182.

algebrizar (Dicc. hist., acep. 2a: 1954, Rey Pastor): 1908, Prom, I, 58.

al-higuí (Dicc. hist., lb: céd. 1918): 1920, LN, 136; 1922, SA, 15.

alineado (Dicc. hist., acep. $2^{\text {a }}$ a 1882, Selgas): 1910, 7 palabras, 68.

amamparar (Dicc. hist.: a 1600?): 1935-1961, Los muertos (1961), 134.

amaricamiento (Dicc. hist.: 1913, J. Ingenieros): 1909, Prom, III, 92.

ambrosino, -a (Dice. hist.: 1528, Delicado y Dicc. Domínguez): 1923, Senos, 71.

\section{ADDENDA:}

abuelo, -a (adj.) (Dicc. hist., 1: 1849): 1912, EL (1920), 288.

acaballar (Dicc. hist., acep. I ${ }^{\mathrm{a}}$ : 1463): 1909, Prom, V, 58.

accidentar (Dice. hist., acep. 3a : 1864, Bécquer): 1911, LM, 126.

aceitazo (Dicc. hist.: 1 ej. apud Pagés y dice. s. XIX): 1923, EN, 384.

acosador (Dicc. hist., acep. 3a : 1888, Galdós): 1931, Ismos, $O C$, t. 2, 1045.

acristalado (Dicc. hist., acep. I ${ }^{\text {a }:}$ 1883-1911, S. Rueda): 1912, Tristán (1913), 74.

acistalar (Dicc. hist., acep. 2a: a 1933, S. Rueda): 1912, Tristán (1913), 74.

aculotar (Dice. hist., acep. $1^{\text {ab }}$ : 1918, Arniches): 1923, Senos, 57.

adamita (Dicc. hist., acep. 4a : 1942, Santamaría): 1923, Senos, 74.

adorno (Dicc. hist., acep. $7^{\text {ab: }} 1956$ Ac.): 1930, ROcc, núm. 88, 27.

amazonismo (Dicc. hist., 1911, Palacio Valdés): 1923, QP (1983), 187.

3. Primeras documentaciones peninsulares, posteriores a uno o más ejemPLOS HISPANOAMERICANOS RECOGIDOS EN DICG. HIST.

ablucionar (1909), Beatriz, 159; 1910, LM, 77 (+2, 1909, 1910).

abracadabra (1908), $M O, 108$.

abracadabrante (1910), 7 palabras, 75; 1911, LM, 144.

abolsar (acep. Ia ) (1923), EN, 214.

abrillantado, -a (acep. 2a): (1935-1961), OF, 154.

abullonar (1911), Tapices, 9; 1923, Senos, 71.

abusivo (acep. $3^{\text {a }}$ ) (1926), ROcc, núm. 34; 'Antología (1955), 337.

acabado (acep. $3^{a}$ ) (1921), La tormenta (1959), 780a.

acordeonista (1935-1961), OF (1961), 146. 
acrata (1908), MO, 125; 1911, LM, 174.

achuras (1934-1943), Lo cursi, 194.

afilalápices (1920), $L N, 200$.

aflautar (1911), Tapices, 41.

afrodisia (1908) MO, 114.

ágape (acep. $1^{\mathrm{a} b}$ ) (1909), Homenaje Fígaro, 50.

agarbanzado (acep. $2^{\mathbf{a}}$ ) (1917), El circo, OC, t. 2, 829.

agrandado, -a (1933), ROcc, núm. 116, 201.

agredir (1910), Prom, XX, 2.

ahuevar (1922), $S A, 74$.

ajedrezado (acep. $\mathrm{I}^{\mathrm{a}} \mathrm{c}$ ) (1929), Efigies, $O C, \mathrm{t} .2,628$.

alambrada (1910), 7 palabras, 69.

alcancía (acep. c) (1942), 'Azorín, OC, t. 1, 1124.

alfiler de gancho (1935-1961), OF (1961), 190.

alfombrado (m) (1912), EL, OC, t. 1, 429.

alharaquiento (en Dicc. hist. todos los ejs. del XX son de Hispanoamérica) (1930), GL, 83.

altiplanicie (1920), $L N, 242$.

alzaprimar (acep. $\left.3^{\text {a }}\right)(1922), S A$.

ambiguo (acep. $2^{\text {a }}$ ) (1923), Senos.

americanizado, -a (la Ac. lo considera exclusivo de América), (1923), $Q P$ (1983) 297.

4. Palabras usadas por Gómez de la Serna en recha anterior a la primera

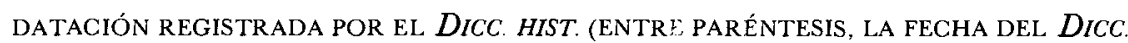
HIST.)

aberrado (1913); 1909, DP (1920), 52; 1910, 7 palabras, 68; Ib., 72.

abismado (acep. $\left.2^{\mathbf{a}} 1941\right) ; 1911$, Tapices (1913), 49.

abortar (acep. ll $^{\mathrm{a} b}$ 1957); 1918, VBN, OC, t. 1, 1384.

abullonado, -a (1928); 1912, EL (1920), 300; 1923, Senos, 110.

aburguesamiento (1920-1922); 1909, Prom, III, 93.

aburguesar ( $\mathrm{I}^{\mathrm{a}}$ acep. 1909; $2^{\mathrm{a}}$ acep. 1957); 1908, MO, 119 (en acepción $2^{\mathrm{a}}$ ). academicista ( $\mathrm{I}^{\mathrm{a}}$ acep. 1924; $2^{\mathrm{a}}$ acep. 1944); 1909, CNL, 12 (en $\mathrm{I}^{\mathrm{a}}$ acep.); 1909 Prom, VII, 90 (en $2^{\text {a }}$ acep.)

acidular (a 1917); 1910, LM (es discutible si se trata de un p.p. o de un adjetivo acidulado, que no figura en Dicc. hist.: "¡Oh, esa (. . .) intercadencia agridulce y acidulada!"'

acera ( $8^{\mathrm{a}}$ acep.: 1919); 1910, $L M, 135$.

acobijo (1946); 1914, ER, OC, t. 1, 164; 1918, Muestrario, 230.

acometivamente (Dice. hist, da 1922 por error: es 1911).

acompañamiento (acep. $6^{\mathrm{a}} \mathrm{b}$ : a 1935); 1918-1924, Pombo, OC, t. 2, 375.

acompasar ( $3^{\text {a }}$ acep.: 1916); 1912, EL (1920), 259.

acromegálico ( $2^{\mathrm{a}}$ acep.: 1935); 1929, ROcc, núm. 74, 237.

acuciador, -a (2ª acep. 1914); 1909, CNL, 21.

adiposo (2 ${ }^{\mathrm{a}}$ acep. 1910); 1908, MO, 33; Ib. 133; 1909, Prom, VI, 91.

aeronaval (1946); 1930, Paris (1986), 183.

aflictivo, $-\mathrm{a}\left(7^{\mathrm{a}}\right.$ acep. 1927); 1920, $L N, 142$.

afroamericano, -a (1937); 1931, Ismos, OC, t. 2, 1053. 
ágape (acep. 2a b: 1910); 1908, MO, 135.

agresivamente (1911); 1909, Prom, VI, 91. agresividad (1910); 1909, DP, 9; CNL, 30 .

aguafiestas (subacepción b.: 1948); 1930, ROcc, núm. 84, 358.

ahogadizo (subacep. d.: 1962); 1918-1924, Pombo, OC, t. 2, 449.

ahuesado, -a (Dicc. hist.: 1912); 1911, Tapices (1913), 75.

aislación (Dicc. hist., por error, da 1922); 1911; 1910, LM, 166 (+5).

ajardinado, -a (1951); 1911, Prom (Tapices, 122).

ajoarriero (subacep. b: 1929); 1921, Banquete Grandmontagne, menú.

ala (20 a acep. 1910 Enciclopedia Espasa); 1910, Prom, XX, 2.

aleteo (4 ${ }^{\text {a }}$ acep. 1926); 1921, La tormenta (1959), 783b.

alfeñicamiento (1931); 1909, Prom, VI, 94.

alforza (acep. la b: 1922); 1911, Moguer (1913), 79.

alígero ( $3^{\text {a }}$ acep.: 1915); 1914, ER, 15.

aliguí (alhiguí) (c1918); 1912, Prom, XXXVII, 136.

alijo (acep. 2a b: 1965); 1923, Ramonismo, 87.

almendrado, -a (dicho de los ojos: 1925); 1912, EL (1920), 261.

alternar ( $7^{\mathrm{a}}$ d.: 1932); 1920, LN, 246 (se encuentra también en 1904 en Baroja, La busca).

aluvión (subacep. g: 1965); 1911, Tapices (1913), 160.

allende (sub. 2c: 1917); 1909, Prom, XI, 64.

ambar (sub. $3^{\text {a }} 1939$ ); 1923, Senos, 58. 